\title{
Conventions of Transcription and Translation
}

$\mathrm{T}$

his book draws heavily from Maya, Nahuatl, and Spanish sources, and all English translations are my own unless stated otherwise. In all instances I strive to make the translations readable and oftentimes favor a figurative rather than a literal translation of the texts. Maya authors did not conform to modern conventions of punctuation, spacing, and paragraphing, so I have included them in the translations. However, to the extent possible I have transcribed the Teabo Manuscript and other documents as they appear in their original form, employing the occasional "..." when water damage or wear renders the text unreadable. For purposes of readability, I employ modern standards for determining line breaks and do not parse the transcription into phrase-segments as is sometimes done to highlight potential poetic devices in the text. The manuscript typically separates paragraphs with a decorative drop cap, and such are represented in the transcription. In instances of possible confusion in the translation, I use parentheses to convey intended meanings and brackets for omitted words. Moreover, in the transcriptions I occasionally comment on the reading of a word, and such are found in the notes.

As is common practice, I employ " $\mathrm{"} \mathrm{for} \mathrm{the} \mathrm{backwards} \mathrm{"c"} \mathrm{used} \mathrm{by} \mathrm{the}$ colonial Maya - today written as " $\mathrm{dz}$ " - and "dz" for those instances where a " $\mathrm{"} \mathrm{appears} \mathrm{in} \mathrm{personal} \mathrm{or} \mathrm{place-names.} \mathrm{Moreover,} \mathrm{the} \mathrm{colonial} \mathrm{Maya} \mathrm{often}$ marked glottal stops with horizontal strokes through "h" and "p," and such are represented here as "ch" and "p". The Maya word yetel, "and or with," often appears abbreviated as a " $y$ " with a horizontal stroke, seen here as " $y$ ". Throughout the main text, Maya, Nahuatl, and Spanish words appear in italics upon their first appearance only. Finally, all biblical citations are from the New American Bible (NAB) as it is employed in English-speaking American Catholic churches today. 
THIS PAGE INTENTIONALLY LEFT BLANK 
THE

TEABO

MANUSCRIPT

a 
o

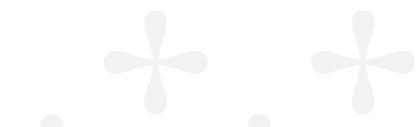

oro

90

0

9

ora

oro

9

9

9

0

9

or

ofo

0

90

o

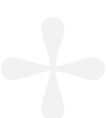

or

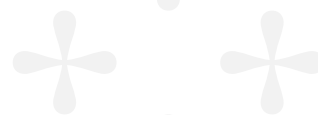

ora

9

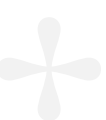

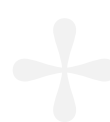

op ofo

\%

0

oro

0

fo of

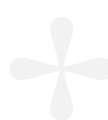

(1)

of

oro

20

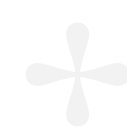

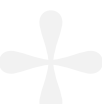

9

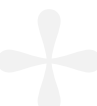

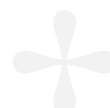

9

of

9

4

0

0

9

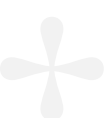

or

0

0

9

0

$f$

0

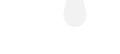

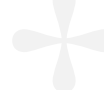

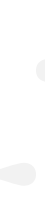

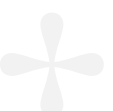

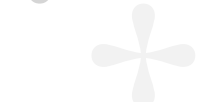

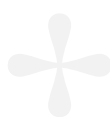

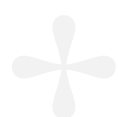

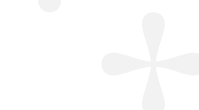

f

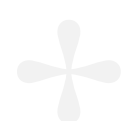

$(x+2)$

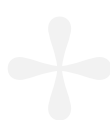

$\frac{1}{2+2}+2$

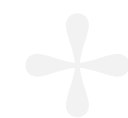

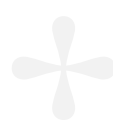

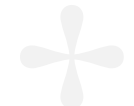

ofo

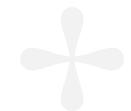

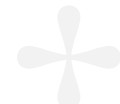

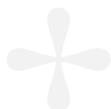

$x^{2}+x^{2}+2$

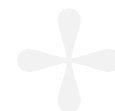

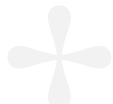

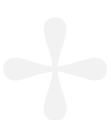

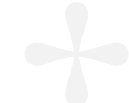

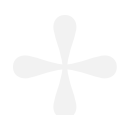

$(1)+1$

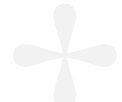

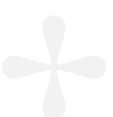

$\left(\frac{10}{2+2}\right.$

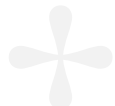

(1) $(2)$

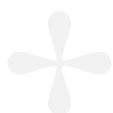

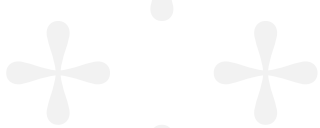

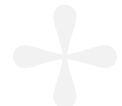

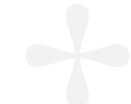

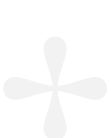

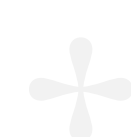

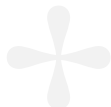

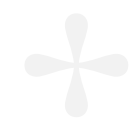

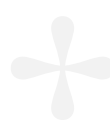

$\frac{1}{2+2}+2$
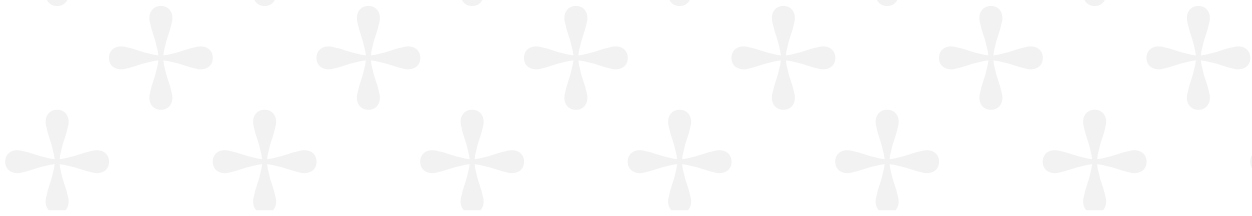